A us der Forschungsgemeinschaft „Das körperbehinderte Kind“ Kö̈ı und dem Psychologischen lustitut der Rheinisehen Friedrich-Milhelms-Liniversität Bonu

\title{
Komplexes Problemlösen bei Jugendlichen mit Hirnfunktionsstörungen ${ }^{3}$
}

\section{Fon Annemarie Fritz und J. Funke}

\section{Einleitung}

Die folgende Arbeit versucht cinen Brückenschlag zwischen der Allgemeinen und der Differentiellen Psychologie vorzunchmen : Das ịn der Allgemeinen Psychologie inzwischen gebräuchliche Kionzept des „Komplexen Problemlösens“ soll mit dem in der Differentiellen Psychologie vielfach beschriebenen Srndrom der "Minimalen Cerebralen Dysfunktion“ (MCD) zusammengebracht werden. Ausgangspunkt hierbei ist die Überlegung, wonach sieb Lernschwierigkeiten von Kindern bzw. Jugendlichen mit MCD ebenfalls beim Bearbeiten komplexer Probleme zeigen sollten. Im Linterschied zu vorliegenden Studien, in denen Intelligenzleistungen von Kindern mit MCD-Symptomatik untersucht wurden, geht diese Arbeit auf jugendliche Probanden ein, bei denen die MCD-Klassifikation bereits länger zurüickliegt.

\section{Wreibung der untersuchungsleitenden Konzepte}

Zunäıhst sollen die Störungen des Lernverhaltens beschrieben werden, die man bei MCD-klassifizierten Kindern beobachten kann. Dabei geht es auch umeine Klarlegung unseres Verständnisses vom Konzept MCD, über das in der Literatur keineswegs Einigrkeit besteht. Weiterhin gehen wir auf die Folgen dieser frühkindlichen Störungen für den weiteren Entwicklungsverlauf im Jugendalter ein. Schließlich wird erläutert, welche Anforderungen beim „Komplexen Problemlösen“ gestellt werden. Vor dem Hintergrund derartiger Überlegungen kann dann die Schilderung der von uns durchgeführten Lntersuchung vorgenommen werden.

Störungen des Lernverhaltens als Kennzeichen der Hirnfunlitionsstörung

Lernschwierigkeiten, die trotz vorhandenex Intelligenzkapazität in Verbindung mit neurologischen Auffälligkeiten, Körperkoordinationsstörungen, Wahrnehmungsstörungen und/oder Konzentrationsschwächen auftraten. wurden vor etwa 20 Jahren zum Konzept der "Minimalen Cerebralen Dysfunktion“ (MCD) oder, den Aspekt der Lembeeinträchtigung stärker betonenden Konzept der „psychoneurologischen Lernschwäche“ (,learning disability" oder ,learning disorder") zusammengefaßt.

Vorrangig war die Annahme, daß es sich bei den beobachteten Schwächen als Folge einer Hirnfunktionsstörung un ein einheitliches Syndrom mit einer gemeinsamen Grund-

1 Die Arbeit wurde durch eine Sachbeihilfe der Deutschen Forschungsgemeinschaft an die Erstautorin (Az. Fr 683/1) gefördert. Wir danken Herm Edgar Erdfelder für kritische Anmerkungen. 
störtung, spezifischen obligaten Symptomen sowie einer einheitlichen Atiologie und Psrchopathologie handelt. Diese Annahme konnte nicht bestätigt werden. Vielmehr zeigte sich, daß eher von einer Vielzahl von Syndromen (vgl. Clements, 1966) bzw. einer Manifestation von Störungen auf unterschiedlichen Ebenen ausgegangen werden muß.

Nach Schmidt und Mitarb. (1984) sowie Esser und Schmidt (1987) kanu sich eine MCD auf drei Ebenen äußern: (1) auf der neurophysiologischen kbene mit Auffälligkeiten im neurologischen und elektroenzephalographischen Bereich, (2) auf der neuropsychologischen Ebene mit visumotorischen Koordinationsstörungen und einer mangelnden Reaktionskontrolle, sowie (3) auf der Ebene dor spezifischen Teilleistungen mit Störungen der sprachlichen Wahrnehmung, der kognitiven Organisation und der visuell diskriminativen Dauerleistung. Dabei umfa B die drilte Ebene eine Vielzahl untersehiedlicher Teilleistungsstörungen, die je verschiedenen funktionsberejehen zugeordnet werden können. Bei aller Unterschiedlichkeit der „Symptombündelungen“, Manifestationsebenen oder Teilleistungsschwächen ist allen Störungen gemeinsam, daß sie in veränderten Lemprozessen zum Ausdruck kommen und bei den Kindern zu umschriebenen Jeistungsausfällen oder allgemeinen Schulschwierigkeiten führen. - In unserer Studie, über die wir weiter unten berichten, bezichen wir uns auf die von Schmidt und Mitarb. (1984) vorgenommene Definition, die auch Grundlage unserer Diagnostik ist, und verwenden zur Kennzeichnung der Population den allgemeinen Begriff der „Hirnfunktionsstörung“.

Erste empirische Untersuchungen zur Erfassung der Lern- und Leistungsprobleme zielten auf eine Analyse der Intelligenz dieser Kinder. Hier fand Schmidt (1972) eine veränderte Leistungsstruktur und folgerte, daß diese Kinder Intelligenzleistungen anders als andere Kinder erbringen. Weitere Lntersuchungen zu der Art des veränderten Lernverhaltens (vgl. Strauss und Kephart, 1955; Meyer-Probst, 1974; Gölnitz und Rösler, 1975; Grüneberg und Remschmidt, 1984) wiesen auf eine geringe Differenzierungsfähigkeit bei der Analyse gestellter Aufgaben und auf eine eingeschränkte Fähigkeit hin, Merkmale nitcinander zu verknüpfen und zu Handlungsketten zusammenzufassen (Integrationsfähigkeit). Spezifische ligenaten in Denken wie Perseverationen (vgl. Cruickshank, 1981) und funktionale Gebundenheit (vgl. Bosch, 1954) verhindern weiter den Erwerb effektiver Problemlösestrategien und verzögern kindliche Lenfortschritte. Besondere Probleme beobachtete man bei der Bewältigung komplexer und abstrakter Aufgaben.

Alle Befunde wurden überwiegend durch Leistnngsminderungen in Subtests von Intelligenz- und Lerntestverfahren bzw. durch Experimente zum Reiz-Reaktions-Lernen und zum Begriffsbildungs-Lernen (vgl. Leyendecker, 1977) gewonneu. Nur selten wurde die Bewältigung komplexer Lernanforderungen durch Verlaufsanalysen beobachtet. Lnseres Wissens existieren kaum gesicherte Aussagen über das Zustandekommen komplexer Problemlöseleistungen.

\section{Einfluß der Störungen auf das Jugendalter}

Übereinstimmung besteht in der Literatur darüber, daß der Aufbau komplexer kognitiver Problemlösefähigkeiten bei hirnfunktionsgestörten Kindern beeinträchtigt ist und die verringerten Fähigkeiten durch motivationale und emotionale l'aktoren wir Mißerfolgsangst, 'Tendenz zur Anstrengungsvermeidung, negatives Selbstkonz'pt rigener Fähig- keiten, Angst zu versagen etc.. noch weiter negativ beeinflußt werden (vgl. Cruickshank, 1981; Esser und Schlack, 1984; Frit\%, 1984). Ob die kognitive Entwicklung dieser Kinder durch die - in der Regel die Schulzeit stark belastende - Schwäche jedoch nachhaltig verändest oder nur vorübergehend verzögert wird, wurde bislang empiriseh wenig untersucht. Allgemein wird wohl davon ausgegangen, dal sich die Schwere der Störungen in der Präadoleszenz und in der Adoleszenz zwar nindert, daß bei Jugendlichen aber inmer noch Schulprobleme sowie Schwierigkeiten im sozialen Kontakt und emotionale Probleme bestehen. Im einzelnen stellte Graichen (1983) in einer Nachuntersuchung an 16jährigen Jugendlichen zum Entwicklungsverlauf von Sprachstörungen fest, „daß sich Teilfunktionsschwächen bis ins Jugendalter nur in geringem Lmfang verlieren “ (S. 360). Danit wird belegt, daß Teilfunktionsschwächen auch im Jugendalter das Leistungsverhalten noch beeinträchtigen.

Sieber und Mitarb. (1984) wiesen in einer Längsschnittstudic an $15 \mathrm{Jahre} \mathrm{alten,} \mathrm{früher}$ unter dem Stichwort „POS“ ("psychoorganisches Syndrom") diagnostizierten Jugendlichen immer noch signifikante Unterschiede in Vergleich zu einer unauffäligen Kontrollgruppe in kognitiven Leistungstests nach. Störungen zeigten sich in den Bereichen Merkfähigkeit, Gedächtnis- und Konzentrationsfähigkeit sowie Gestalterfassung und -wiedergabe.

Hopkins (1979) wies zwar eine Verringerung der Impulsivität nach, stellte aber fest, daß den Jugendlichen Handlungsprogramme und Strategien zur Problemlösung fehlten.

Aus amerikanischen Lüngsschnittstudien zum Entwicklungsverlauf der Störungen läßt sich ebenfalls der Befund ableiten (vgl. die Übersicht bei Minde und Steinhausen, 1982), daß weiterhin visumotorische Koordinationsstörungen, deutliche Auffälligkeiten im Bereich der Aufmerksamkeitssteuerung und der kognitiven Organisation sowie Beeinträchtigungen des Selbstwertgefühls und Störungen des sozialen Verständnisses und der sozialen Anpassung bestehen.

Übereinstimmend weisen die Untersuchungsbefunde auf das Fortbestehen einer gestörten kognitiven Leistungsfähigkeit im Jugendalter hin. Diese hat sich allerdings nicht in gravierenden Entwicklungsdefiziten manifestiert, sondern ist in ihrer Ausprägung sogar geringer geworden. Einschränkend müssen diese Ergebnisse jedoch auf die Überprüfung basaler Funktionen sowie den Bereich von Intelligenztestmessungen, Tests zur Erfassung kognitiver Impulsivität und Interferenzneigung bezogen werden, so daß auch hier kaum Aussagen über die Fähigkeit der Jugendlichen zum komplexen Denken möglich werden. Untersuchungen, in denen die Fähigkeiten von Kindern und Jugendlichen mit MCD-Symptomatik beim Lösen komplexer Probleme eruiert wurden, liegen jedoch bislang nicht vor.

Anforderungen durch komplexe Probleme

Die in den vergangenen Jahren neu entwickelten Instrumente zur Erfassung der „operativen Intelligenz" (vgl. Dörner, 1986) stammen aus dem Bereich der Problemlöseforschung. Sie verlangen vom Probanden $(\mathrm{Pb})$ den aktiven Umgang nit einem unbekannten dynamischen Simulationsszenario in unterschiedlich realistischen Einbettungen (z. B. Raumfahrt, Politik, Ökonomie). Eigenschaften derartiger Problemstellungen sind: (1) die 
Komplexität der Aufgabenstellung, vielfach festgemacht an der Anzahl beteiligter lïnflußgrößen; (2) die Velnetztheit dieser Variablen mitereinander; (3) die Intransparanz der Situation, auch hinsichtlich des zu erreichenden Zieles; (4) die Eigendynamik des Systems, also stine eigenst ändige Entwicklung über dic Zeit hinweg auch ohne äuberes Zutun. Die Anforderungen, die in derartigen Situationen an den Pb gestellt werden, unterscheiden sich somit in vielfältiger Weise von denjenigen, die herkömmliche Verfahren der Intelligenzdiagnostik verlangen. Ungeklärt ist allerdings, wofür der Lmgang mit jeweils neu konstruierten Szenarien ein Indikator sein soll und welche konkreten diagnostischen Informationen aus dem Lingang eines $\mathrm{Pbn}$ mit cinem computersimulierten System ableikbar sind. Die Ablcitung von Indikatoren bereitet insbesondere dann Sehwierigkeiten, wemn das Simulationssystem keine objektive Gütebestimmung zuläßt (vgl. die Kritik hicran bei Funke, 1986). Ein Ausweg besteht darin, kleine und überschaubare Szenarien mit wohlbekannten Eigenschaften zu verwenden, bei denen sich die genannten Schwierigkeiten nicht ergeben. Dieser Weg wird in der vorliegenden Arbeit beschritten.

In Hinblick auf die MCD-Symptomatik ist zu konstatieren, daß die vielfäligen Teilleistungsschwächen sich nicht nur in globalen Minderleistungen bei der Systembearbeitung niederschlagen sollten, sondern auch zu speziellen Phänomenen ( $x$. B. Perseverationen) Anlaß geben könnten. Darauf wird bei der Schilderung der Hy pothesen näher eingegangen.

\section{Besehreibung der Untersuchung}

Vor dem Hintergrund der eben diskutierten Problemalik der Leistungsdefizite von MCD. klassifizierten Kindern wurde eine empirische Lntersuchung geplant, in der eine Gruppe von Jugendlichen mit MCD-Symptomatik hinsichtlich ihrer l.eistumg beim Bearbeiten eines komplexen Problems verglichen werden sollte mit einer Kontrollgruppe. Die nachfolgenden Abschnitte beschreiben das Lntersuchungsmaterial, die Stichprobe sowie die Hypothesen.

Untersuchungsmaterial, Instrultion und Versuchsdurchführung

Als Untersuchungsmaterial diente eine Variante des sog. "Ökosystems", das in der Arbeit von Funke (1985) genauer beschrieben und hier nur in Kürze referiert wird. Von den sechs bei Funke (1985) beschriebenen Varianten kam die einfachste Form, "OEKOLL" genannt, zur Anwendung. Das Sinulationsszenario "OLKOLL“ ist ein lineares Kleinsystem bestehend aus drei exogenen und drei endogenen Variablen, das den Pb in die Rolle eines Kleingärtners versetzt, der einen Giarten zu betreten hat. Vit den drei exogenen Variablen "Gift", "Schädlingsfresser" und "Dünger" (= drei der Waßnahmevariablen) kimn der Pb Einfluß auf dic drei endogenen Variablen "Kufer", "Wasserverschmutzung" und "Blätterzahl" (=die drei Züstandsvariablen) nehmen. Folgende, Strukturgleichungen liegen dem System zugrunde, wobei die in Klammern stehenden Angaben die zeitliche Abhängigkeit verdeutlichen:
(1)
$\mathrm{K}(t+1)=0,9 \times \mathrm{K}(t)+1 \times \mathrm{S}(t)$
$\mathrm{W}(t+1)=1 \times \mathrm{W}(t)-0,1 \times \mathrm{G}(t)$
$\mathrm{B}(t+1)=1 \times \mathrm{B}(t)+10 \times \mathrm{D}(t)$

Das durch die Gleichungen (1) bis (3) beschriebene System ist vergleichsweise einfach: Der jeweils nächste Zustandswert einer Variablen - also zum Zeitpunkt $t+1$ - hängt zum einen vou ihrem eigenen vorherigen Zustand zu Zeitpunkt $t$ ab (wobei nur bei (1) ein von den Faktor 1 abweichendes Gewicht gewählt wurde), zum anderen von genau einer der Eingriffsvariablen zum Zeitpunkt $t$ (wobei die Gewichte für S, Ci und D so gewählt wurden, daß keine komplizierten Rechenoperationen erforderlich waren). Weder $\mathrm{Zu}$ fallseffekte: noch Zeitverögerungen erschwerten die Bearbeitung. Die Startwerte für $\mathrm{K}$, W und B betrugen 200, 10 und 5000, die Zielwerte, deren Erreichung im letzten von insgesamt fünf Jurchgängen gefordert wurde, betrugen 200, 0 und 10000. Je 1)urchgang standen sieben lingriffstakte zur Verfügung.

In der schriftlich gegebenen Instruktion wird die Künstlichkeit des Simulationssystems betont und der $\mathrm{Pb}$ zur freien Exploration der Wirkzusammenhänge aufgefordert. Zugleich wird erläutert, daß vor der ersten Systembearbeitung sowie im Anschluß an jeden der insgesant fünf Durchgänge mit je sechs Takten ein sog. „Kausaldiagramm" erstellt werden soll, in dem der $\mathrm{Pb}$ in graphischer Form die von ihm bereits erkannten bzw. vermuteten Zusammenhänge zwischen den Variablen aufzeichnen sollte. Dic ersten vier Durchgänge werden als Erkundungsphase bezeichnet (,Wissenserwerbsphase“), in denen der $\mathrm{Pb}$ Eingriffe nach eigenen Vorstellungen tätigen kann. In folgenden fünften Durchgang wird die Erreichung des, bereits zu Beginn mitgeteilten Zielvektors verlangt (Wissensanwendungsphase").

Die Versuchsdurchführung șah vor', daß das beschriebene Simulationsspiel in den Rahmen einer umfangreichen Diagnostik eingebettet war. Die Datenerhebung erfolgte im Frühjahr und Sommer 1986 in Einzelsitzungen jeweils in Anwesenheit einer Versuchsleiterin2. Nach Aushändigung der. Instruktion und dem Ausfüllen des ersten Kausaldiagramms konnte mit der eigenständigen Bearbeitung des Simulationsspiels begonnen werden. Der $\mathrm{Pb}$ durfte selbständig mit einem Tischcomputer interagieren, indem er seine gewünschten Eingriffe via Tastatur eingab und die erzielten Zustandsänderungen auf dem Bildschirm angezeigt bekam. Dic zurückliegenden Zustände und Eingriffe des jeweiligen Durchgangs blieben auf dem Bildschirm zur Gedïchtnisentlastung präsent. Der Pb unterlag bei seinen Explorationen keinerlei Zeitbeschränkungen. Notizen durften gemacht werden. Zwischen jedem der fünf Durchgänge wurden fünf Fragen zur Motivation des $\mathrm{Pb}$ gestellt sowie die Kausalmodelle mit den subjektiven Annahmen über die Variablenvernetzung erhoben.

\section{Stichprobe}

An der Untersuchung nahmen insgesamt 53 Jugendliche im Alter von 14;3-16;8 Jahren teil. 3/1 Jugendliche stammen aus einer Gesamtpopulation sog. "Risikokinder" ( $N=444)$ des Jahrgangs 1970/71, die in zwei Kölner Kliniken geboren wurden und Schwangerschafts(z. B. Infektionen, Bluthochdruck der Mutter) bzw. Geburtsrisiken (z. B. operative Entbindung, Mehrlingsgeburt, Nabelschnurkomplikationen) aufwiesen. Bei ihnen sollte im

2 Für dic sorgfältige Durchführung der Untersuchung bedanken wir uns bei Frau stud. päd. Elena Höhner, die diese Studie als studentische Hilfskraft betreut hat. 
Rahmen einer prospektiven Studie ülerprüft werden, inwieweit derarlige Risikofaktoren eine Hirnfunktionsstörung bedingen. ${ }^{3}$ Für 70 dieser Kinder konnte zun Zeitpunkt des Schuleintritts - also 1976/7 - durch Summationsdiagnose (vgl. Lempp, 1980) die Manifestation einer Hirnfunktionsstörung mit hoher Wahrscheinlichkeit festgestellt werden. Sie wiesen Probleme in der Gesuntkörperkoordiuation. Wahroehmungsstörungen und Beeinträchtigungen in spezifischen kognitiven Funktionen bei einer insgesamt guten Gesamtintelligenz auf. Eine Nachuntersuchung der Pbu nach den Kriterien von Schmidt und Mitarb. (1984; zweite und dritte Manifestationsebene) ergab eine Zuordnung von 12 Kindern zur neuropsvchologischen Fbene und 48 Kindern zur Ebene der spezifischen Teilleistungen, $10 \mathrm{Kinder}$ zeigten Überschneidungen auf beiden Ebenen. Für 5 Kinder konnte die ursprüngliche Diagnose nicht bestätigt werden.

Weitere Untersuchungen dieser Pbn fanden 1980 und 1982 statt. Hier warden Leistungsergebnisse zum Lern- und Problemlöseverhalten sowic zum Motivationsverlauf der hirnfunktionsgestörten Kinder mit denen einer nach Alter, Geschlecht und IQ parallelisierten Kontrollgruppe verglichen (vgl. Fritz, 1984). - An der jetzigen Cntersuchung haben insgesamt 34 der prospektiv erfaßten „Risikokinder" und 19 Jugendliche der Kontrollgruppe teilgenommen.

Un zu überprüfen, ob die Jugendlithen immer noch Symptome im Sinne einer MCD aufweisen, wurden sie 1986 erneut test psvehologisch untersucht. Für die MCD-Diagnose wurden - wic bereits oben erwähnt - die Parameter der zweiten und dritten Ebene der MehrebenentFalldefitition von Schmidt übernommen. Nach dieser diagnostischen Einteilung zeigten von den 34 Jugendlichen 15 immer noch Auffäligkeiten, davon sechs im neuropsychologischen Bereich und acht auf der Ebene der Teilleistungsschwäche. Eine Vp war auf beiden Ebenen auffällig. Bei den restlichen $19 \mathrm{Pbn}$ ließ sich die Diagnose MCD mit den verwendeten Mebinstrumenten nicht mehr erhärten. Dieser Befund stimmt weitgehend mit dem schulischen Lern- und Leistungsverhalten der Jugendlichen überein: Bei den Pbn, bei denen eine Hirnfunktionsstörung erneut diagnostiziert werden konnte, bestehen auch aktucll noch Lernprobleme und schulische Schwierigkeiten, während sich bei den anderen Jugendlichen das Leistungsverhalten weitgehend stabilisiert hat.

Für die anschließend durchgeführten Gruppenvergleiche stehen nach ibzug von insgesamt sechs infolge. Datenausfällen nicht verwertbaren $\mathrm{Pb}_{n}$ somit drei verschiedene Stichproben zur Verfügung: (1) die Pbn mit stabiler MCD-Symptomatik $\left(\mathrm{N}=14 ;,, M C D^{i c}\right)$, (2) die Pbn mit ehemaliger MCD-Klassifikation, die sich 1986 nicht bestätigen ließ und die daher nur als potentielle MCD-Pbu betrachtet werden sollten $\left(\mathrm{N}=17 ;\right.$, $\left.\mathrm{POT}^{\prime}\right)$, sowie (3) die $\mathrm{Pbn}$ der Kontrollgruppe $\left(\mathrm{N}=16 ;, \mathrm{KON}^{*}\right)$.

\section{Hypothesen}

Die von uns formulierten Erwartungen beziehen sich allesamt auf die Schwierigkeiten hirnfunktionsgestörter Kinder beim Lösen komplexer Probleme. Ihre Begründung erfahren diese Erwartungen einerseits durch die eingangs beschriebenen Störungsebenen,

3 Einer älnnlichen Fragestellung wird in der „Rostocker Längsschnittuntersuchung“ (vgl. Meyer-Probst und Teichmann, 1984) nachgegangen. auf denen die MCD-Befinition aufbaut, andererseits durch die in einzelnen Stadien nachgewiesenen Lernschwierigkeiten. Dic Probleme hirnfunktionsgestörter Jugendlicher - so die Hypotheseu - sollten sich in folgenden wit II1 bis Hö bezeichneten Bereichen niederschlagen, über deren genauc Erfassung anschließend berichtet wird:

(H1) Die vorgegebenc Zielerreichung in der Anwendungsphase der Srstembearbeitung erfolgt verlangsant, es resultiert ein größerer Zielabstand.

(H2) Die Vielfalt der verwendeten Strategien beim. Eingreifen in das System ist verriugert.

(H3) Es liegt eine geringere Abstraktionsfähigkeit im Sinne unangemessenerer Vorstellungen über die Variablenbeziehungeu vor. Deren Ursache kann auch eine geringere Fähigkeit zur Erfahrungsauswertung sein.

(H4) Die Lernfähigkeit, wie sie sich im Verlauf der fünf bearbeiteten Durchgänge zeigt, ist verlangsamt.

(H5) Der Einfluß nicht-kognitiver Variablen läßt sich in form geringerer Anstrengungsbereitschaft und höherer Mißerfolgsangst nachweisen.

Die fünf Hypothesen beziehen sich auf Unterschiede der MCD-Gruppe in Vergleich zur Kontrollgruppe; die potentielle MCD-Gruppe sollte bezüglich ihrer Kennwerte eine intermediäre Stellung einnehınen.

\section{Beschreibung der abhängigen Variablen}

Für die eben beschriebenen Hypothesen müssen Indikatoren angegeben werden, die die jeweiligen Konstrukte in der Empirie verankern. Dabei wird auf Kennwerte bezug genommen, wie sie beim Bearbeiten dynamischer Systeme üblicherweise anfallen. Generell erwarten wir für die $\mathrm{Pbn}$ mit Hirnfunktionsstörungen diesbezüglich verschlechterte Leistungen.

Prinzipiell stehen Daten aus zwei verschiedenen Bereichen zur Yerfügung: Zum einen liegen Systemdaten vor, die die Eingriffe des $\mathrm{Pbn}$ in das System sowie die Reaktion des Systems auf diese Eingriffe darstellen. Zum anderen liegen die vor dem ersten und nach jedem der fünf Durchgänge angefertigten Kausaldiagramme vor, die im Vergleich mit der tatsächlich vorliegenden Kausalstruktur eine mögliche Annäherung der subjektiven Vorstellungen an die objektiven Bedingungen dokumentieren können. Auf beide Datenquellen wird bei der nachfolgenden Schilderung der abhängigen Variablen eingegangen.

Die in $\mathrm{H} 1$ erwähnte Verlangsamung der Zielannäherung sowie die größere Ziełdistanz ergibt sich aus der Bearbeitung des fünften Durchgangs : Über das Euklidiscle Abstandsma $\beta^{4}$ wird die zu jedem Takt vorliegende Distanz zum geforderten Zielvektor bestimmt. Dessen Verlauf sowie dessen mittlerer Wert stellen dic Daten dar, auf die diese Hypothese bezogen werden soll.

Die mit $\mathrm{H} 2$ angesprochene geringe Vielfalt an Eingriffsstrategien wird festgemacht an der Verteilung insgesamt vorkommender $0-, 1$-, 2- und 3-fach-Eingriffe. Als 0-Eingriff zählt

4 Dieses $M a ß$ hat sich in bisherigen Studien als brauchbares Abstandsmaß erwiesen. Für die Wahl anderer Metriken gibt es keine inhaltlichen Aryumente.

12 z. Psychologie $196-2$ 
ein Takt, in dem der $\mathrm{Pb}$ bei keiner der drei Kingriffswariablen cinen Wert ungleich Null eingibt; chin 3-fach-lingriff bedeutet dagegen einen Eingriff in alte Variablen gleichreitig.

Die in It3 genannte geringere Abstraktionsfähigke:it wird an der Qualität der ersteflten Kausaldiagramme abgelesen. Hierfür wurden Punkte nitch eincm festgelegten Schema vergeben, die zwischen Null (= keine richtige Beziehnng) und Eins (= alle Beziehungen erkinnt) schwankten. Um cin möglichst ,faires" $\mathrm{M}_{11} 13$ der Repräsentationsgüte zu erhalten, wurde dieser GS-Index nach dem bei Jiunke (J985, S. 456) beschriebenen Verfahren ermittelt.

Die unter H5 erwartete Verlangsamung des Lernens soll durch die Differenz zwischen der Qualität des Kausaldiagramms im ersten und im Ietzten Durchgang erfaß3t werden. Ist diese gering, könnte dies für einen geringen Wissenszuwachs im Sinne der Hypothese sprechen.

Die in $\mathrm{H}_{4}$ angesprochene geringere Anstrengungsbereitschaft und höhere Mißerfolgsangst wird mit den entsprechenden liragen des nach jedem Durchgang vorgelegten Motivationsfragebogens erhoben. Ausgewertet wurden die Antworten auf achtstufig skalierte Fragen, wie sie vor dem zweiten, dritten, vierten und fünften I)urchgang den Pbu zur Bearbeitung vorgelegt wurden. Aus diesen Angaben wurde jeweils ein Summenscore für die jeweilige Variable bestimm , der zwischen 0 und 28 liegen konnte.

\section{Darstellung der Ergebnisse}

Mit HI wurde eine Verlangsannung der Zielannäherung sowie im letzlen Durchyang eine größere Zieldistanz. für die MCD-Gruppe prognostiziert. Tảbelle I enthält die enklidischen Distanzwerte der jeweiligen Systemnektoren zum Zielvektor eimmal über die sechs Takte hinweg, zum anderen in bezug auf die drei zu steuernden Y. Yariablen (alle Daten stammen aus dem fünften Bearbeitungsdurchgang).

Ohne inferenzstat ist ische Prüfung (vgl. die Höbe und Heterogenit ät der Streunngen) wird die Ilypothese einer bei VICD-Jugendlichen verlangsamten Zielerreichung verworfen : Die miluleren Abstände sind über alle Takte hinweg kleiner als die Distanzen der beiden Refe-

Tah. 1.a Mittlere Euklidische Distanzen $(x)$ und deren Streuung $(s)$ awischen System- und Zielvektor, (a) über Takte sowie (b) über Variablen, getrennt nach den drei (iruppes

\begin{tabular}{lccrrrrr}
\hline \multirow{2}{*}{ Gruppe } & \multicolumn{2}{c}{ Abweichung taktweise } & \multicolumn{1}{c}{} \\
\cline { 2 - 8 } & & Takt 1 & Takt 2 & \multicolumn{1}{c}{ Takt 3 } & Takt 4 & \multicolumn{1}{c}{ Takt 5 } & Takt 6 \\
\hline KON & $x$ & 3264,2 & 4356,9 & 5185,1 & 5010,6 & 5430,4 & 4486,4 \\
(N=16) & $s$ & 4946,8 & 8082,6 & 10135,2 & 10780,1 & 11249,5 & 11026,4 \\
POT & $x$ & 2786,5 & 3517,0 & 13892,3 & 13741,0 & 33871,3 & 140182,8 \\
(N=17) & $s$ & 4654,9 & 7301,5 & 52699,6 & 52752,9 & 94239,2 & 485377,8 \\
MCD & $x$ & 1347,5 & 1424,4 & 1154,7 & 1102,4 & 954,6 & 2906,1 \\
(N=14) & $s$ & 584,4 & 1093,0 & 1156,9 & 1340,5 & 1042,2 & 7452,7 \\
\hline
\end{tabular}

Tab. I. b

\begin{tabular}{|c|c|c|c|c|}
\hline \multirow{2}{*}{ Gruppe } & & \multicolumn{3}{|c|}{ Abweichung variablen weise } \\
\hline & & $Y_{1}$ & $Y_{2}$ & $Y_{3}$ \\
\hline KON & $x$ & 92,2 & 217,7 & 13556,9 \\
\hline$(N=16)$ & $s$ & 167,5 & 832,7 & 24878,9 \\
\hline POT & $x$ & 263,5 & 33,7 & 103701,1 \\
\hline$(N=17)$ & $s$ & 679,7 & 105,7 & 330645,1 \\
\hline $\mathrm{MCD}$ & $x$ & 568,1 & 7,7 & 3869,4 \\
\hline$(\mathbf{N}=1 / 4)$ & $s$ & 1863,1 & 7,5 & 4508,0 \\
\hline
\end{tabular}

renzgruppen, bis auf zwei Ausnahmen (von Takt 1 auf 2 sowie von Takt 5 auf 6 ) nehmen die Distanzen sogar ab. Bei den beiden übrigen Gruppen nehmen diese Distanzen in der Regel zu. Auch bezogen auf die drei zu steuernden Variablen (vgl. rechten Teil von Tab. I) errichen die Pbn der MCD-Gruppe für $\mathrm{Y}_{2}$ und $\mathrm{Y}_{3}$ die geringsten mittleren Distanzen.

Dieser anf den ersten Blick überraschende Befund einer besseren Bearbeitung des Systens durch di: MCI)-Gruppe erweist sich jedoch insofern als trugschlüssig, als die AV untersehiedlich „heftiges" Eingreifen in das System reflektiert: Zählt man nämlich aus, wieviel Pbn den vorgegebenen Zielvektor simultan in mindestens einem Takt erreich haben, sind dies 19\% der KON-, 12\% der POT- jedoch 0\% der MCD-Gruppe!

Die unter $\mathrm{H} 2$ postulierte geringere Vielfalt unterschiedlicher Fingriffsstrategien bei MCDJugendlichen kanı nicht in dieser Form behauptet werden. Tabelle II zeigt die mittleren Häufigkeiten unterschiedlicher Eingriffsarten in das System für die drei Probandengruppen.

Zwar zeigt sich von der 'Tendenz her ein Bild, das in Linklang mit unseren lirwartungen steht, insofern als die XCD-Gruppe die geringste Verwendung von diagnostisch aufschluBreichen 0- bzw. Ifach-Eingriffen und die stärkste Verwendung von 3fach-Eingriffen machı, jedoch läßt sich nur für LGI diese Aussage statistisch absichern, wenn man von der in diesem Fall gegebenen Varianzheterogenität absieht.

Tab. 1I. Mittelwerte $(x)$ und Streumgen $(s)$ des Vorkommens vou Eingrifien auf keiner, einer, zwe oder allen drei exogenen Variablen (EGO, EG1, EG2 und EG 3), getrennt nach den drei Gruppen, sowie Errebnisse der F-Tests

\begin{tabular}{llllrr}
\hline \multirow{2}{*}{ Gruppe } & \multicolumn{2}{l}{ Eingriffsart } & & \\
\cline { 3 - 5 } & & EGO & EG 1 & EG 2 & EG 3 \\
\hline KON & $x$ & 0,93 & 9,33 & 8,00 & 11,73 \\
$(\mathrm{~N}=15)$ & $s$ & 0,96 & 9,35 & 5,84 & 9,39 \\
POT & $x$ & 0,65 & 7,53 & 9,23 & 12,59 \\
$(\mathrm{~N}=17)$ & $s$ & 0,93 & 5,57 & 4,94 & 8,19 \\
MCD & $x$ & 0,43 & 3,86 & 9,14 & 16,57 \\
$(\mathrm{~N}=14)$ & $s$ & 0,76 & 3,50 & 5,91 & $\mathbf{7 , 2 8}$ \\
\hline $\mathrm{F}(2,43)$ & 1,17 & $2,57 *$ & 0,23 & $\mathbf{1 , 3 8}$ \\
\hline
\end{tabular}

* $p \leqq 0,10$, bei inhomogenen Variauzen 
In bezug auf die mangelnde statistische Stülzung gilt ähnliches für $11 \%$, mit der eine geringere Abstraktionsfähigkcit der MCl)-Gruppe (gemessen an der Qualität der erstellten Kausaldiagramme) vorhergesagt wurde, aber nicht belegt werden kann. Tab. 3 enthält die entsprechendeu Bewertungen für drei unterschiedliche, logisch voneinander abhängige „Wissensformen“, die sich von Relations- über Richtungs- hin zu Stärkewissen durch wachsende Präzision auszeichnen.

Tab. III. Mittlere Punktwerte $(x)$ und de'ren Streuung $(s)$ für drei unterschiedlich strenge Bewertungen der erstellten Kausaldiagramme, getrennt nach den drei Gruppen, sowie Ergebnisse der F-Tests

\begin{tabular}{|c|c|c|c|c|}
\hline \multirow{2}{*}{\multicolumn{2}{|c|}{ Gruppe }} & \multicolumn{3}{|c|}{ Wissensform } \\
\hline & & Relation & Richtung & Stärke \\
\hline $\mathrm{KON}$ & $x$ & 1,07 & 0,63 & 0,37 \\
\hline$(\mathrm{N}=16)$ & $s$ & $0,2 \pi$ & 0,60 & 0,48 \\
\hline POT & $x$ & 0,95 & 0,44 & 0,16 \\
\hline$(\mathrm{N}=17)$ & $s$ & 0,31 & 0,35 & 0,31 \\
\hline MCD & $x$ & 1,00 & 0,42 & 0,16 \\
\hline$(\mathrm{N}=14)$ & $s$ & 0,37 & 0,47 & 0,28 \\
\hline$F(2,44)$ & & 0,51 & 0,88 & 1,81 \\
\hline
\end{tabular}

Wiederum nur tendenziell erkennt man Lnterschiede in der erwarteten Richtung: Mit wachsender Anforderung an die Genauigkeit der Kausaldiagramme erzielen die Mitglieder der MCD-Gruppe schlechtere Werte. Interessant daran ist, daß die POT-Gruppe fast den gleichen Abfall aufweist und sich damit zwar von der KON-Gruppe abhebt, nicht aber von der MCD-Gruppe.

Verlangsamter Wissenserwerb, ausgedrückt als Differenz des im Kausaldiagramm erhobenen Systems vor und nach Bearbeitung des Systems, wurde durch H4 der MCD. Gruppe vorhergesagt. Auch die Prognose läßt sich statistisch nicht untermauern, wenngleich für aile Wissensformen die MCD-Gruppe einen geringeren Zuwachs verzeichnet als die KON-Gruppe (vgl, Tab. IV).

In dieser Hinsicht unterscheiden sich POT- und MCD-Gruppe nicht voneinander.

Tab. IV. Mittelwerte $(x)$ und Streuungen $(s)$ des Zugewinns von Versuchsbeginn zu Versuchsende in bezug auf drei Wissensformen, getrennt nach den drei Gruppen, sowie Ergebnisse der F-Tests

\begin{tabular}{lllll}
\hline \multirow{2}{*}{ Gruppe } & & \multicolumn{2}{l}{ Wissensform } & \\
\cline { 3 - 5 } & & Relation & Richtung & Stärke \\
\hline KON & $x$ & 0,06 & 0,11 & 0,09 \\
$(\mathrm{~N}=16) s$ & 0,07 & 0,11 & 0,11 \\
POT $\quad x$ & 0,04 & 0,08 & 0,06 \\
$(\mathrm{~N}=17)$ & $s$ & 0,09 & 0,09 & 0,11 \\
MCD $\quad x$ & 0,04 & 0,08 & 0,05 \\
$\mathrm{~N}=14)$ & $s$ & 0,10 & 0,10 & 0,08 \\
\hline F $(2,44)$ & 0,23 & 0,32 & 0,75 \\
\hline
\end{tabular}

Mit 145 wurden nicht-kognitive EinfluBgrößen als Differenzierungsnerkmale von KONInd .ICD-(iruppe erwartet. Jie VCD-Gruppe solle danach geringere Anstrengungsbereitschaft und höhere Hißerfolgsangst aufweisen. Wie Tabelle V zeigt, läß sich der erste Teil dieser Aussage wiederum nur tendenziell aufrechterhalken, der zweite Teil dagegen nicht.

Tab. V. Mittelwerte $(x)$ und Strentungen (s) für vie.r motivationale Variallen, getrennt nach den drei Gruppen, sowie Errobnisse der F-Tests

\begin{tabular}{|c|c|c|c|c|c|c|c|}
\hline \multirow{2}{*}{ Variable } & \multicolumn{2}{|l|}{$\mathrm{KON}$} & \multicolumn{2}{|l|}{ POT } & \multicolumn{2}{|l|}{$\triangle I C D$} & \multirow{2}{*}{$F(2,44)$} \\
\hline & $x$ & $\bar{s}$ & $\bar{x}$ & $s$ & $x$ & $s$ & \\
\hline Ans & 15,94 & $5,9 / 4$ & 15,53 & 6,30 & 12,86 & 6,58 & 1,05 \\
\hline Scli & 15,25 & 6,34 & $15, \overline{3} 3$ & 5,62 & 10,71 & 6,17 & $2,96^{*}$ \\
\hline Erf & 15,25 & 6,17 & 13,59 & 5,68 & 16,36 & $5,5 \overline{5}$ & 0,88 \\
\hline $\mathrm{MiB}$ & 5,19 & 7,92 & 6,18 & 5,09 & 5,93 & 6,32 & 0,10 \\
\hline
\end{tabular}

Summenwerte aus viermal vorgeterten Fragen mit achtstufiger Skalierung. Ans = Anstrengungs -

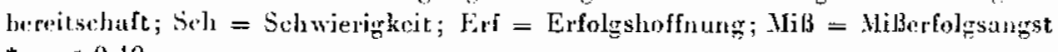
* $p \cong 0,10$

Statistisch bedeutsam ist die unterschiedliche Einschätzung der Aufgabensehwierigkeit: KON- und POT-Gruppe schätzen diese gle ichermaßen höher ein als die .ICD-Gruppe, der die Problemstellung insgesamt leichter erscheint.

Unabhängig von den hypothesenberogenen Auswertungen gab es einige interessante Phänomene, deren Vorkommen in den drei Gruppen registriert wurde und hier mitgeteilt werden soll. Bei diesen Phänomenen handelt es sich im einzelnen um folgendes

(1) Die Verwendung positiver wie negativer Zahlenwerte als Eingaben bei den exogenen Viariablen.

(2) Das Vorkommen von Reversionen, d. b. der Rücknahne eines eben getätigten lïngriffs durch einen Eingriff gleicher Starke, aber gegenläufiger Richtung.

(3) Das Vorkommen von Fehlanalysen, d. h. dem Festhahen an ersichtich falschen Hypothesen.

(4) Das Verfolgen individueller Zielwerte unabhängrig von den rom Versuchsheiter gematchten Vorgaben.

Tab. VI. Häufigkeit des Vorkommens lustinmenter Ereignisse: in absolnten Zahlen sowie in Prozent der jewciligen Gruppe

\begin{tabular}{|c|c|c|c|c|c|c|}
\hline \multirow{2}{*}{ Ereignis } & \multicolumn{3}{|c|}{ absolute Zaliler } & \multicolumn{3}{|c|}{ Prozent } \\
\hline & $\overline{\mathrm{KON}}$ & POT & XICD & KON & POT & $\overline{\mathrm{MCD}}$ \\
\hline negative Zahlen & 11 & 12 & 9 & 68,7 & 70,6 & 64,3 \\
\hline Reversionen & 7 & 4 & 9 & 43,7 & 23,5 & 64,3 \\
\hline Fehlanialysen & 1 & 2 & 1 & 6,2 & 11,8 & 7,1 \\
\hline jndiv. Ziele & 6 & 4 & 7 & 37,5 & 23,5 & 50,0 \\
\hline xogen $=$ endogen & 5 & 5 & 4 & 31,2 & 29,4 & 28,6 \\
\hline
\end{tabular}

* $p \leq 0,10$ 
(5) Die Verwendung der Werte endogener Variablen als Werte für die Eingriffsvariablen, d. h. die Zustandswerte des Taktes $t$ werden als Eingabewerte des Taktes $t+1$ übernommen.

Das Vorkommen dieser $S$ pezialfälle sowohl in absoluten Häufigkeiten als auch prozentual in bezug auf dit jeweilige Gruppe zeigt Tabelle VI.

Vergleicht man die Daten der MCD-Gruppe aus Tabelle 11 mit denen der beiden anderen Gruppen, sind allenfalls die bäufigeren Vorkommmisse von Reversionen und individuellen Zielwerten bemerkenswert. Wegen der geringen Fallzahlen wurden keine statistischen Tests durchgefülı't.

\section{Diskussion}

Grundlage der vorliegenden Untersuchung ist eine Analyse des komplexen Problemlöseverhaltens von Jugendlichen, bei denen in Alter von sechs Jahren eine MCD-Diagnose gestellt worden ist. Die Überprüfung der Diagnose bej den Pbn im Alter von 15 Jahren zeigte in Übereinstimnung mit Befunden aus der Literatur (vgl. Schmidt und Drömann, 1986) eine Bestätigung der Diagnose bei $44 \%$ der Pbn, bei $56 \%$ der Pbn ließ sich die Diagnose demgegenüber nicht mehr erhärten.

Obwohl sich in der Gruppe der wiederholt als hirnfunktionsgestört diagnostizierten Jugendlichen Pbn mit unterschiedlichen Teilleistungsschwächen befanden, gingen wir von der Hypothese aus, daß sich die - immer noch nachweisbaren - Beeinträchtigungen in basalen Funktionen ( $z$. B. Störungen der serialen Integration, der visuell diskriminativen Dauerleistungen, der Reaktionskontrolle und der Daueraufmerksamkeit) negativ auf den Aufbau komplexer Problemlösefähigkeiten bzw. hindernd für den Frwerb effektiver Problemlösestrategien auswirken sollten.

Von den Pbn mit nicht replizierbarer Diagnose wurde erwartet, daß sie bezüglich ihrer Kennwerte eine intermediäre Stellung einnehmen. Denn obwohl Leistungsschwächen durch Nachreifung und Intervention im Entwicklungsverlauf ausgeglichen werden körnen, gingen wir davon aus, daß im Alter von 15 Jahren nocb kejevellständige Kompensation für abstrakte Aufgaben höherer Komplexität stattgefunden hat. Dieser Befund würde die Aunahme stützen, daß Beeinträchtigungen in einzelnen Teilleistungen nicht nur zu umschriebenen Teilleistungsschwächen führen, sondern talsächlich „breitere“, den allgemeinen Lernprozeß beeinträchtigende Auswirkungen haben.

Die oben dargestellten Ergebnisse entsprechen nicht den Erwartungen. Hierfür mögen zwei Ursachen verantwortlich sein: (1) die Hypothesen über die Auswirkungeı von Hirnfunktionsstörungen auf die Problemlösefähigkeit sind nichı stichhaltig, (2) die gewählten Indikatoren komplexen Problemlösens erweisen sich als untauglich, derarlige Unterschiede zu reflektieren. Hierüber kanm nicht entschieden werden. Die folgenden Ausführungen verstehen sich daher als Interpretationsvorschlag für das berichtete Ergebuismuster unter der Annahme, daß die diagnostischen Systenkennwerte valide Indikatoren des komplexen Problernlösens darstellen. Sie sind nicht zwingend durch die Befunde im pliziert. Erst weitere experimentelle Untersuchungen können die sicher plausiblen Sicht. weisen zu empirisch tragfähigen Aussagen machen.

\section{Problemlöseverlauf der MCD-(iruppe}

Bei dem vorgegebenen Problem handelt es sich um ein fiktives Kleinsystem mit einer bestinmten Anzahl exogener und endogener Variablen, deren Wirkweise durch den Lmgang mit dem System erkannt werden soll. Die Ausgangsbedingungen sind für alle Pbn gleich: Sie müssen in einem ihnen unbekannten System L̈rfahrungen sammeln und diese Erfahrungen in einer nachfolgend gestellten Aufgäbe gezielt einsetzen.

Die KON- und POT-Giruppe sammelt diese Erfahrungen überwiegend durch 1- und 2fachEingriffe in das System. Jurch diese Bedingungsanalyse der Merkmale wird ein Wissenserwerb ermöglicht, der die sofortige Rückfühlunger der neu entstandenen Zustandswerte aul die vorangegangene Intervention zuläßt.

Demgegenüber erfolgt der Wissenserwerb der MCD-Cruppe überwiegend durch multivariate, alle drei Variablen gleichzeitig betreffende Eingriffe. Dieses scheinbar ,komplexe" Vorgehen verschaff den Pbn jedoch keine Informationen über die Wirkweise der einzehen Variablen in System und läß die Bedeutung (Richtung und Stärke) der Variablen unaufgedeckl. Ein solches Vorgehen kanu als Ausdruck einer mangelnden Differenzierungsfähigkeit der Phrt interpretiert werden: Sie sind nicht in der Lage, dio Merkmale des Systems gegeneinander abzugrenzen und deren Bedingungsgröß̧en systematisch zu erarbeiten. Es gelingt ihnen keine "Orduungsbildung durch Merkmalsdiskriminierung" (Meyer-Probst, 1974), die ihnen einen zielgerichteten Wissenserwerb ermöglichen würde. Infolgedessen bleibt ihr Wissen über das System ungenau und möglicherweise fehlerhaft, da falsche Hypothesen über angenommene Zusammenhänge durch diese Art der Intervention kitum aulgedeckt werden könuen.

Das als Folge dieses Vorgehens geringere Wissen der MCD-Gruppe über die Zusammenhänge der Variablen im System spiegelt auch die (allerdings nicht signifikante) geringere Qualität der Kausaldiagramme wider. Veranlaßt zur Erfahrungsauswertung zeigt sich, daß die MCD-Gruppe erwartungsgemäß weniger und vor allem weniger exakte Informationen über die Wirkweise des Systems erworben hat bzw. nicht in der Lage ist, die erworbenen Informationen zu strukturieren und dic Zusammenhänge zwischen den Merkmalen herzustellen. Denn Voraussetzung für die Erstellung von Kausaldiagranmen ist neben ciner korrekten Merkmalsinalyse auch die Fähigkeil, die Merkmale in ein Bedingungsgefüge zu integrieren (Integrationsfähigkeit). Die mangelnde tähigkeit zur Differenzierung und Integration der Merkmale wird auch in dem (allerdings nicht signifikanten) verlangsamten Lernzuwachs der MCD-Gruppeüber die Zusammenhänge im Systen deutlich. Hinsichtlich des Lösungserfolgs für dit Problemstellung zeigt sich zwar kein Lnterschied zwischen den Cruppen, sic unterscheiden sich jedoch hirsichtlich der angewendeten Strategie, die Lösung zu erreichen. Da die KOX- und POT-Gruppe hinreichende Informationen über die Wirkweise der einzclnen Variablen und deren Verknüpfung untereinander gesammelt haben, sind sie auch in der Jage, in der Wissensanwendungsphase mehrere Variablen gleichzeitig zu steuern und so simultan den Zielzustand anzustreben. Die MCDGruppe, die demgegenüber nur erschwert luformationen über einzelne Variablen erhalten hat und zwischen diesen weniger gut Bezichungen herstellen konnte, kann daher auch die Steuerung des Systems nur in einzeluen Schritten vornehmen. 
Die Ergebnisse weisen darauf hin, da 13 die allgemein als Differenzierungs- und Intrgrationsschwäche beschriebene kognitive Leistungsbeeinträchtigung himfunktionsgestörler Kinder (vgl. Fritz, 1986) auch im Jugendalter noch forlbesteht und eine effektive Erirbrit ung komplexer Probleme behindert.

\section{Problemlöseverlauf der POT-Gruppe}

Die Pbn der POT-Gruppe gahen bei der Beabeitung des Problems in gleicher Weise wie die Pbn der KON-Gruppe vor. Ihre Problembearbeitung beginnt mit der für ein planvolles Handeln notwendigen Analyse der Werkmale und Bedingungen des Handlungsfeldes. Durch 1- und 2-fach-Eingriffe nehmen sie eine sorgfältige Verkmalsanalyse vor und sammeln Erfahrungen über die Bedeutung der einzelnen Variallen. Die eine günstige? Problembearbeitung beeinträchtigende Differenzierungsschwäche der MCD-Gruppe scheint bei den Jugendlichen dieser Gruppe nicht mehr vorzuliegen.

Die durch ihre Strategie gewonnenen Erfahrungen können jedoch von den Pbn der POT Gruppe entweder nicht in optimaler. Weise ausgewertet werden (mangelnde Fähigkeit zur Erfahrungsauswertung) oder die abslrahierten Erfahrungen können nicht aufeinander bezogen werden, d. h. die Merkmale können nicht zu cinem Bedingungsgefüge von untereinander in Beziehung stehenden Variablen verknüpft werden (mangelnde Integrationsfähigkeit). Denn bei der Cbertragung der Informationen sinkt die Qualität der Kausaldiagramme ebenso ab wie bei dev ,.VCD“-Gruppe. Daß es sich hierbei um eine Schwäehe der Pbn handelt, die nachhaltig dic Qualität und Effektivität des Problemlösprozesses beeinträchligt, verdeutlicht auch das Ergebnis zum Verlauf des Wissensorwerbs über die fünf Durchgänge hinweg. Insbesondere bei der präzisen Kenmzeichnung der „Winflußstärke", den die exogenen Variablen auf den Systemzustand nehmen, ist der lernzuwachs über das Wissen der Pbn nur gering.

Trotz dieser dem Kausaldiagramm zufolge wenig integrierten Erfahrungen bemühen sich auch die Pbn der POT-Gruppe - allerdings in geringerem Ausmaß als die KON-Cruppe in der Wissensanwendungsphase um eine Sicuerung des Gesamtsystems. Dieser Befund läßt vermuten, daß die Pbn durch ihr Vorgehen der elementaren Merkmalsanalyso auch Erfahrungen über die Zusanmenhänge der Variablen sammeln, die nachfolgend in strategiebezogenes Handeln einfließen. Sie sind jedoch nicht in der Lage, das Wissen über die im System bestehenden Beziehungen auf ein abstrahiertes Modell æu übertragen.

Motipationsverlauf

Die subjektive Einschälzung der Pbn über ihre Lrwartung, das Systern zu erkennen, und über ihre Angst, bei dieser Aufgabe zu versagen, erbrachte keinen Lnterschied zwischen den Gruppen. Alle drei Gruppen sind zuversichtlich im Hinblick darauf, die Lösung zu finden, und befürchten vicht, zu versagen.

Allerdings schätzen sie die Schwierigkeit der Aulgabe unterschiedlich eir. Die VCDGruppe hält diese Aufgabe für signifikant leichter als die beiden anderen Gruppen, entsprechend schätzl sie den Anstrengungsaufwand, der für diese Aufgabe zu erbringen ist, geringer ein (Ergebnisse nux' in den' 'Tendenz). Eine derartige Einschätzung der Aufgabenschwierigkeit ist auf dem IJintergrund der bei den Jugendlichen dieser Gruppe auch

aktuell noch bestehenden Lemprobleme und Schulschwierigkiten zu sehen. So haben die Jugentlichen dieser Gruppe über viele Jahre hinweg Miferfolge in Jeistungssituationen erfahren, olne offeklive Strategien zu deren Vermeidung oder Bewäligung gefunden zu haben. Giauben Jugendliche mit diesen Erfahrungen, eine Aufgabe bewältigen zu kömen, so ist es nahegelegt, daß sie den Läsungserfolg nicht in ilnren Fähigkeiten, sondern in der geringen Schwierigkeit der Aufgabe sehen. Line Aufgabe, die keine hohen Anforderungen stellt, kamm aber auch nicht die Investition hoher Anstrengungen erforderlich machen.

Dir Kombination dieser Aussagen weist darauf hin, da B die Jugendlichen dieser Gruppe nitht über ein fähigkeitsbezogenes Solbstkonzept verfügen, sondern immer noch (vgl. Frity, 19S f) mißerfolgsorientiert die erfolgreiche Bewäligung von Aufgaben einer geringen Aufgabenschwierigkeit zuscheiben.

In Conerschied zu dieser Gruppe schützt die Cruppe der themals als MCD diagnostizierten Jugendlichen ihre Erfolgserwartung, die Aufgabensehwierigkeit und ihre Anstrengumgsbereitschaft in gleicher Weise ein wie die Kontrollgruppe. Beide Gruppen sind zuversichtlich, auch eine als sehwierig eingeschälzle Aufgabe bewäligen zu können. Diese Jugendlichen haben demzufolge ein erfolgszuversichtliches Selbstkonzept. eigener Fähigkeiten.

Für die Jugendlichen der POT-Gruppe bedeutet dies, daß die Stabilisierung ihres Leistungsverhaltens mit ciner Stabilisierung ihrer Motivationslage einherging.

\section{A usblick}

Der Veryleich zwischen den drei Lntersuchungsgruppen läßt vermuten, daß sie drei unterschiedliche „Entwicklungsstufen“ repräscntieren: Im Vergleich zur KON-Gruppe besitzt die MCD-Gruppe eine ausgeprägte Differenzierungs- und Integrationsschwäche, die POT-Gruppe bezieht in ihre Strategie Differenzicrung mit tin, aber der „nächsthöhere" komplementäre Prozeß der Integration, d. h. die flüssige Abwicklung abstrakter Aufgaben ist noch nicht voll ausgeprägt. Die Ergebnisse sprechen jedoch für eine sukzessive Annäherung an die altersgleiche Ciruppe. Die Diagnose VCD erweist sich sonit als keineswegs „,umabänderliches Schicksal“, wenngleich die Ursachen für cine Verbesserung von Leistungsschwächen in dieser Arbeit nicht aufgezeigt werden komnten.

Gleichzeitig bestätigt sich, daß 'Teilleistungsschwächen Auswirkungen auf die Bearbeitung komplexer Probleme haben. Dabei stellt sich auch dic Frage nach der Trainierbarkeit entsprechender Fähigkeiten, auf die hier nicht nähcr eingegangen werden kann.

Abschließend bleibt festzuhalten, daß wegen der vergleichsweise geringen Fallzahlen in dieser Pilotstudie nur stärkere Effekte nachweisbar waren. Nachfolgende Lintersuchungen mit umfangreicheren Stichproben können sicherlich noch differenziertere Befundmuster eruieren. Die Aualyse des komplexen Problemlöseverhallens bei Pbn mit Hirnfunktionsstörungen erweist sich dann als fruchtbar, wenn spezifische Defizitc, aber auch spezifische Kompensationsleistungen mit einem derartigen Zugang sichtbar gemacht werden können. 


\section{Zusammenfassung}

Ausgehend von Uberlegungen zum lionzept der "Mitimalen Cerebralen Dysfunktion" (MCD) wird gef'agt, in welcher Weise jugendliche Prohanden mit MCD-Symptomatik bei der Bearbeitung „komplexer Problenne“ vorgehen. Hierzu wurden insgesamt 53 Pbri einer prospektiven Längsschnittuntersuehung im Alter von $15 \mathrm{~J}$ ahren mit einem computersinulierten komplexen Problem konfrontiert. Die Pbn wurden in drei Gruppen eingeteilt: 14 Pbn wiesen eiue stalbile MCD-Symptomatik and („,MCD"),

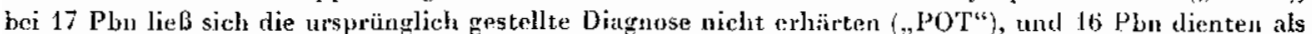
Kontrollgrupłe $\left(, \mathrm{KON}^{\mathrm{N}}\right)$. Im Problemlöseverlauf zeigt sich, dab die MCD-Gruppe üler gexinge Differenzierungs- und mangelnde Integrationsfihigkeit verfügt. Die Problembearlocitung dieser Cruppe ist motivatioual durch eine Mißerfolgsorientiertung grekennzeichuet. Die POT'Gruppe unterscheidet sich von der KON-Gruppe durch eine noch vorliegende Integrationsschwitche. Neben liragen nach der Brauchlarkeit des nenartigen diagnostischen Zugiungs zu Teilleistungsseliwiichen beschäftigt sich die abschlieBende Diskassion mit dem Problen der Stabilität von MCD-Synptonatiken in Entwicklungsverlaur.

\section{Summary}

The study investigates complex problem solving in young individuals aged 15 having "minimal cerebrat dysfunctions". Following up on the considerations concerning the concept of "Minimal Cerebral Dysfunctions" (MCD), the question, how adolescent subjects with the MCD-symptomatic would go about dealing with "eomplex problems", was raised. For this purpose a total of 53 subjects, each 15 years old, from a prospective longitudinal study, were confronted with a computer-simulated complex problem. The subjects were split into three groups: 14 subjects displayed a stable MCD-symptomatic ("MCD"), 17 subjects did not confirm the original diagnosis ("POT"), and 16 suljects acted as a control group ("CON"). During the problem-solving the MCD-gronp showed that they had insufficient discriminatory and deficient integrational ablities. This groups treat ment of the problem is characterized, motivationally speaking, by their failare orientation. The POT-group differed from the KON-group with respect to their still noticeable integrational weakuess. Besides questioning the usefulness of the novel diagnostical approach to partial performance debility the concluding disenssion deals with the problem of the stability of $\mathrm{MCO}$-characteristies in the course of development.

\section{Резюме}

На основе обсуждения концепта "минимальной церебральной дисфункции "ставится вопрос о том, какие стратегии применяют юные испытуемые с симлтоматикой минимальной цере брально дисфункции при реп ении комплексных проблем. Для әтого в исследовании по методу продольного среза 53 испытуемым 15-летнего возраста были предложены проблемы, симулированные на компьютере. Испытуемые делились на три группы: у 14 испытуемых об̆наруживалась стабильная симптоматнка минимальной церебральной дисфункции, у 17 испытуемых не удалось подтвердить ранее поставленный диагноз ("РОТ"), а 16 испытуемых

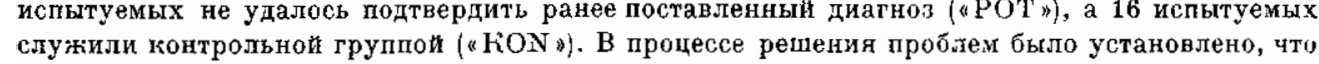
испытуемые с минимальной церебральной дисфункцией обладают женьшими способностями к дифференциации и интеграции Разработка проблем в этой группе мотивационно харагтеризуется ориентировкой на неуспех. Группа "РОT " отличается от группы " заметной слабостью интеграционных способностей. Наряду с вопросами применения нового диагностического подхода $\mathrm{K}$ частичным нарушениям умственных способностей в заключительной дискуссии обсуждается проблема стабильности симптоматики минимальной цере бральной дисфункции в процессе развития.

\section{Literatur}

Bosch, G. P'sychopathologic der kindlichen Hirnschädigung. Bericht über die Literatur von 19/0-1953. Fortsehritle der Neurologie, Psychiatrie 22 (1954) 425-456.

Clements, S. D.: Minimal brain dysfunction in children. NTNDB Monograph 3 (LSPIS Publication 1415). Washington: U.S. Department of Health, Education and Welfare 1966.

Cruickshank, W. M.: Sehwierige Kinder in Schule und Elternhaus. Berlin: Marhold 1981.

Dörner, D. Diaknostils der aperativen Intelligen\%. Diagnostica 32 (1986) 290-308.

Esser, O.; Schlack, H. G.: Berateng bei Minimaler zerebraler Dysfunktion. Rehabilitation 23 (1981) XVII-XXIV:

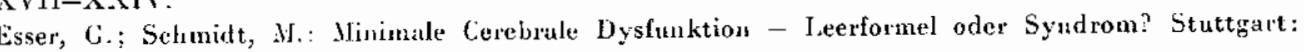
F. binke 1987 .

Fritz, A.: Kognitive und motivationale Lrsachen der Lernschwiche von Kindern mit einex minimalen cerebralen Dysfunktion. Beriin: Marhold 198́.

Fritz, 1.: Erfolyreicher im Lerneil. Ein Fürderprogtamm für le rnschwache Schüler. Berlin : Marhoid 1986. Funke, J.: Steuerung dynamischer Systeme dureh Aufbau und Anwendung subjektiver Kausalmodelle. Z. Psychol. 193 (1985) $435-457$.

Funke, J.: Komplexes Problemlösen. Bestandsaufualune und Perspektiven. Heidelberg: SipringerVerlitg 1986.

Gölnitz, G.; Rösier, H.-D. (Hrsg.): Psychologische E'ntersuchungen zur Entwicklung hirngeschäddigter Kiuder. Berlin: Deutscher Verlag der Wissenschaften 1975.

Graichen, J.: Verschwindeu Teilfunktionssch wächen? Z. Finder- und Jugend psychiat. 11 (1983) 355-362. Grüuteberg, B.; Remselımidt, H.: Störungen der sozialen Wahrnehmung bei Kindem mit Minimaler Cerebraler Dysfunktion. Z. Kinder- und Jugendpsychiat. 1 (1984) 33-52.

Hopkius, J.: Cognitive style in adults originally diagnosed as hyperactives. J. Child Psychol. and Psychiat. $20(1979) 209-216$.

Lempp, R. Orauische Psychosyndrome. In: Lehrluch der speziellen Kinder- und Jugendpsychiatrie. Hrsy.: Harbater. H.: lempp, R.; Nissen, G.; Strunk, P. Berlin: Springer-Verlag 1980.

Leyendecker, C.: Lernverhalten behinderter Kinder. Eine vergleichende experimentelle Lintersuchung zum Lernverhalten bei Kindern mit cerebralen Bewegungsstörungen. Rheinstetten: Schindele 1977. Meyer-Probst, B.: Üer kognitive Leislungsveränderungen hirngeschädigter Kinder. Z. Psychol. 182 (1977) $181-2$ 11.

Meyer-Probst, B.; Teichmann, H.: Misiken für die Persönlichkeitsentwicklung im Kindesalter. Leipzig: G. Thieme 1984.

Minde, K. Steinhausen, H.-C.: Die laugrristige Entwicklung konzentrationsgestörter und lyyperaktiver Kinder. In: Das konzentratiousgestörte und liyperiktive Kind. Hrsg.: Steinhausen, H.-C. Stuttgart: Kohlliammer 1982. S. 180-190.

Schmidt, M. II.; Kinder nit cerelralen Beweğngsstörungen in ilırem intelligenten Verhalten. Berlin: Marhold 1972.

Sehmilt, M. Il.: Drörnaun, S. (Hrsg.): Langzeitverlauf kinder- und jugendpsychiatrischer Erkrankiungenl. Stuttgart: F. Enke 1986.

Schmidt, M. H.; Esser, G.; Allehoff, W.; Geisel, B.; Laucht, M.; Reichert, W. J.; Woeruer, W.; Voll, R.: Syndromcharakter und Bedeutung cerebraler Dysfunktion in Abhängigkeit von Falldefinition und Bezugspopulation. Suarländisches Ärzteblatt 3 (1981) 225-241.

Sieber, M.; Haas, J.: Hain. P.; Spirig, C.; Corboz, R.: Verschwinden die Beeinträehtigungen leicht hirn(1984) 12-22. Strauss, A.; kephart, N.: Psychopathology and education of the brain-injured child. New York: Grune \& Stratton 1955 .

Eingegangen ims September 1987

Ansehr. d. Verf.: Dr. J. Funke

Psychologisches Institut der Universität Bonn Römerstr. 164, D - 5300 Bonn 